D.O.I.: $10.3895 / \mathrm{S} 1808-04482009000400008$

\title{
ANÁLISE DE UMA OPERAÇÃO LOGÍSTICA DE CARREGAMENTO E EXPEDIÇÃO DE CIMENTO POR SIMULAÇÃO COMPUTACIONAL
}

\section{ANALISYS OF CEMENT LOADING AND DISPATCHING LOGISTIC OPERATION BY COMPUTER SIMULATION}

\author{
Miguel Afonso Sellitto ${ }^{1}$; Miriam Borchardt ${ }^{2}$; Giancarlo Medeiros Pereira ${ }^{3}$ \\ ${ }^{1}$ Universidade do Vale do Rio dos Sinos - UNISINOS - São Leopoldo - Brasil \\ sellitto@unisinos.br \\ ${ }^{2}$ Universidade do Vale do Rio dos Sinos - UNISINOS - São Leopoldo - Brasil \\ miriamb@unisinos.br \\ ${ }^{3}$ Universidade do Vale do Rio dos Sinos - UNISINOS - São Leopoldo - Brasil \\ gian@unisinos.br
}

\begin{abstract}
Resumo
Este artigo descreve uma aplicação de simulação computacional por planilha eletrônica, cujo objetivo foi identificar a melhor alternativa para redução de tempo de permanência de caminhões em um sistema de filas de uma operação logística. A operação é o carregamento e expedição de cimento ensacado. Os veículos chegam à portaria e entram em fila para autorização de carregamento. Liberado para carga, o veículo aguarda ser requisitado pela operação, que ocorre em duas baias de carregamento. São admitidos quarto veículos por baia, mantendo controlado um inventário de oito veículos dentro da operação. Uma baia carrega a partir de estoque, com mais alto desempenho, outra carrega direto da ensacagem. No periodo das 6:00 às 8:30 da manhã a taxa de chegada supera a taxa de saida, gerando fila crescente. O problema é encontrar uma alternativa de reorganização do processo que reduza o tempo médio de permanência, sem penalizar excessivamente a ocupação dos recursos. Três alternativas propostas foram testadas em um modelo de simulação em planilha. Elementos da teoria das filas e o diagrama de resultados foram usados para avaliar o resultado da simulação. A melhor alternativa foi a reativação, por duas horas e meia por dia, de uma terceira baia manual, atualmente desativada. O tempo médio de permanência passou de 144 para 91 minutos.
\end{abstract}

Palavras-chave: Simulação com planilha, Simulação em operações logísticas, Teoria das filas em logística, Diagrama de resultados.

\section{Introdução}


A competitividade na fabricação tem sido determinada, ao longo do tempo, pelo custo e desempenho dos produtos manufaturados. Atualmente, porém, compostos de produtos mais variados, prazos de entrega mais curtos e confiabilidade na entrega passaram a ter importância na competição. Com isto, passou-se a exigir das estratégias de produção que ofereçam, simultaneamente, redução nos tempos até a entrega e nos custos de produção. Surgiu então um trade-off: grandes lotes e inspeção 100\% têm garantido, respectivamente, redução de custos e garantia de qualidade, mas acarretam compostos com menos variedade, tempos de atravessamento maiores e mais variabilidade nas datas de entrega. Tempo de atravessamento curto facilita o cumprimento das datas-devidas, atendendo um objetivo do mercado. Carregamento pleno e previsível aumenta a ocupação dos recursos produtivos e reduz o custo, atendendo um objetivo da fábrica (WIENDAHL, 1995).

Incerteza na demanda, variabilidade nas trocas de lotes e desempenho baixo nas cadeias produtivas e logísticas são fatores que dificultam a redução de custo e colocam em risco o cumprimento de datas-devidas. Sob certas circunstâncias, os objetivos logísticos de clientes e de fabricantes podem ser contraditórios, sendo necessária uma solução de compromisso: uma configuração tal que reduza os tempos de atravessamento sem prejudicar demasiadamente a ocupação do sistema produtivo. Nos casos em que há preparação de máquinas, ao se aumentar o lote de produção, reduzindo a perda por preparação, aumenta-se o inventário em processo, criando uma fila de espera para as ordens, o que retarda o atendimento aos novos pedidos. Ao diminuir o lote, por variabilidade nas entradas, é possível que baixe a ocupação da fábrica.

$\mathrm{Na}$ indústria cimenteira, o transporte é feito por veículos de carga. Em lotes maiores, a ocupação do equipamento é alta, mas a espera pelo próximo lote reduz a ocupação dos veículos. Em lotes menores, a ocupação do veículo cresce, mas, devido à variabilidade, cresce o risco de faltar veículo na hora da carga, reduzindo a ocupação do equipamento. Uma técnica que pode ser útil em casos como o descrito, em que há trade-offs entre alternativas e a variabilidade é significativa, é a simulação computacional. Por esta técnica, é possível testar diversas alternativas, avaliando-se seus potenciais resultados. Como a técnica emprega ambientes virtuais de análise, sem necessidade de alteração de arranjos físicos, necessitam-se baixos investimentos.

O objetivo deste artigo é apresentar uma pesquisa envolvendo simulação de alternativas para a organização da logística de expedição de uma fábrica de cimento, entregue ensacado e a granel, por caminhão e trem. A pesquisa se delimita à expedição de cimento ensacado por caminhões. O objetivo de pesquisa é encontrar uma alternativa que reduza o tempo médio de espera de clientes por carga. Não é objetivo contextualizar, aprofundar ou revisar a simulação computacional como técnica de pesquisa, haja vista sua presença na literatura de engenharia de produção. A questão de pesquisa é: que alternativa de organização dos recursos de expedição da 
fábrica reduz mais o tempo de espera de clientes e qual a repercussão da alternativa no uso dos recursos e no custo de expedição? O método de pesquisa é a simulação computacional para a análise das alternativas. Este trabalho continua e complementa Sellitto (1999), que usou uma abordagem similar para a logística de produção interna da mesma fábrica.

Entre muitas outras, seguem algumas aplicações da simulação na solução de problemas logísticos. Persson e Araldi (2007) integraram o modelo SCOR de gestão de cadeias de suprimentos e uma ferramenta de simulação de eventos discretos para análise de efeitos dinâmicos. Chatfield et al. (2006) desenvolveram um simulador de operações em cadeias de suprimentos. Terzi e Cavalieri (2004) fizeram uma revisão de artigos investigando o uso de simulação na gestão de cadeias de suprimentos (SCM). Disney et al. (1997) usaram simulação para modelar grandezas logísticas, tais como inventário e tempo de atravessamento. Zee e Vorst (2005) propuseram um quadro de trabalho para o uso de modelagem e simulação em logística.

O restante do artigo está organizado em: referencial teórico necessário para a pesquisa, incluindo filas e simulação computacional em operações logísticas, a pesquisa e discussão sobre a alternativa apontada pela simulação.

\section{Filas em operação logística}

Um processo de filas surge em uma operação logística quando chegam tarefas e, por variabilidade, em um dado instante, o número de tarefas que já chegaram é maior do que o de recursos disponíveis. Os intervalos entre as chegadas das tarefas e o tempo de processamento no recurso produtivo são variáveis aleatórias. O processo estará em equilíbrio se as taxas médias de chegada e saída forem iguais durante o tempo da análise (PAPADOPOULOS et al., 1993). Um processo de filas em expedição de caminhões consiste em uma ordem de carga que chega a uma operação de carga, espera sua vez na fila, é processada e é despachada. A ordem está sujeita à troca de prioridades e interrupções por manutenção ou falta de materiais.

Um sistema básico de fila é representado pelos parâmetros $\mathrm{A} / \mathrm{B} / \mathrm{m} / \mathrm{K} / \mathrm{M}$, respectivamente: distribuições de probabilidade do tempo entre chegadas e do tempo de serviço, número de recursos, capacidade de serviço e número de clientes potencial. Para capacidade infinita de serviço, número de clientes infinitos e disciplina FIFO (First-in First-out), a notação torna-se A/B/m. Para número de chegadas por unidade de tempo seguindo a distribuição de Poisson (por conseqüência, os intervalos de tempo entre as chegadas seguem a distribuição exponencial negativa) e tempos de serviço seguindo a exponencial negativa, o sistema torna-se $\mathrm{M} / \mathrm{M} / 1$. A letra $\mathrm{M}$ vem de processo markoviano (ou de Poisson). Os requisitos teóricos de tal processo são: (i) o número de ocorrências em intervalos disjuntos é independente; (ii) o número de ocorrências em um intervalo de tempo 
depende apenas do tamanho do intervalo; e (iii) em um intervalo de tempo suficientemente pequeno, a chance de duas ocorrências simultâneas é negligenciável (PIDD, 1998; HILLIER; LIEBERMAN, 1995).

Uma fila em operação logística de carga pode ser analisada segundo a Figura 1.

Figura 1: Fila em operação logística

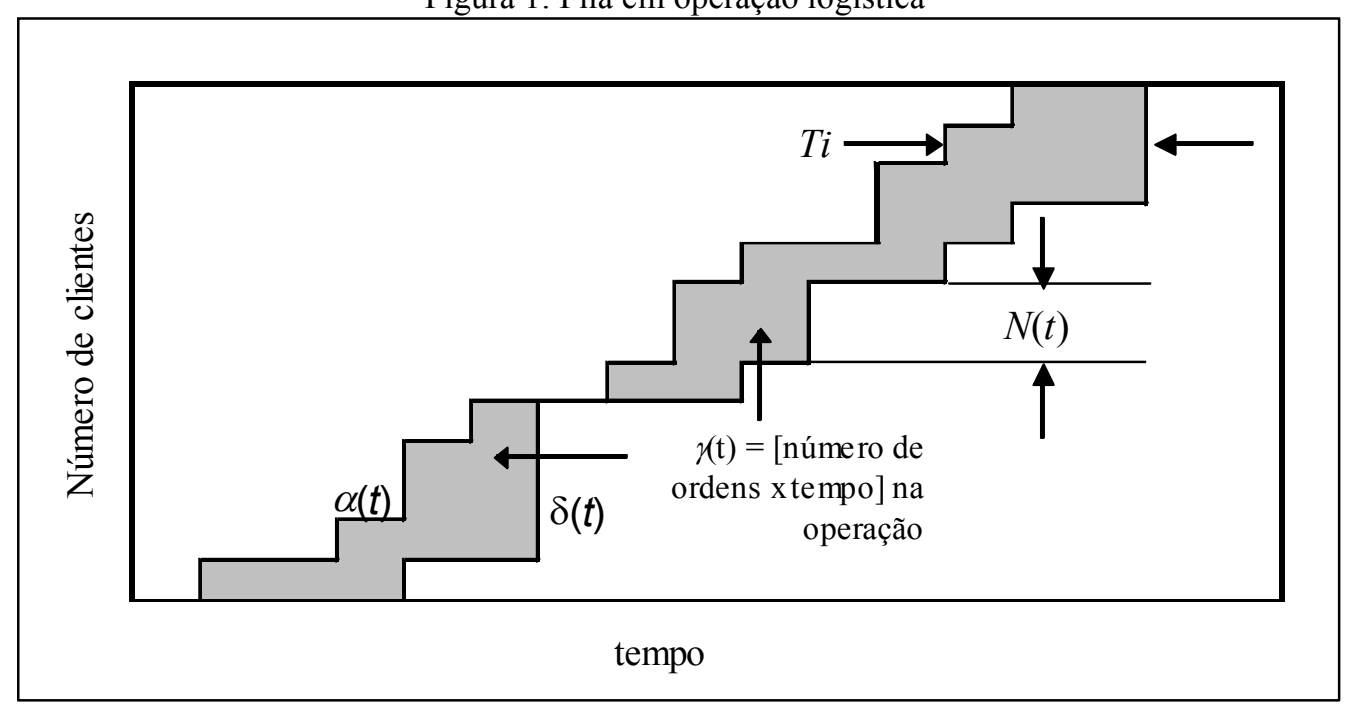

fonte: Kleinrock (1975)

Sejam $\alpha(t)$ e $\delta(t)$ os números de chegadas e saídas de ordens de carga no intervalo $[0, t]$. O número de ordens $N(t)$ na operação é dado por (1). A área $\chi(t)$ entre os traçados representa a permanência [ordens.unidade de tempo] das ordens na operação em $[0, t]$. A taxa média de chegada $\lambda$, o tempo médio $T_{m}$ gasto por ordem e o número médio de ordens $N_{m}$ na operação em $[0, t]$ são dados por (2), (3) e (4). Chega-se a (5), a lei de Little. A ocupação do sistema $\rho$ é dada por (6), na qual $\mu$ é a taxa média potencial de saída de ordens por unidade de tempo. Se $\rho<1$, o sistema é estável e o tamanho da fila converge a um valor finito. Se o sistema é M/M/1, os tempos médios de espera e os números médios de ordens (e veículos) na fila e total no sistema são dados por (7), (8), (9) e (10) (KLEINROCK, 1975; HILLIER; LIEBERMAN, 1995). 


$$
\begin{gathered}
N(t)=\alpha(t)-\delta(t) \\
\lambda=\alpha(t) / t \\
T_{m}=\gamma(2) / \alpha(t) \\
N_{m}=\gamma(t) / t \\
N_{m}=\lambda T_{m} \\
\rho=\lambda / \mu \quad(6) \\
T_{q}=\lambda /[\mu(\mu-\lambda)] \\
T_{m}=1 /(\mu-\lambda) \\
N_{q}=\lambda^{2} / \mu(\mu-\lambda) \\
N_{m}=\lambda /(\mu-\lambda)
\end{gathered}
$$

Se o tempo de atendimento seguir uma distribuição generalista, o sistema torna-se M/G/1 ( $\sigma=$ desvio-padrão e $\mu=$ média do tempo de processamento) e valem de (11) a (14) (HILLIER; LIEBERMAN, 1995):

$$
\begin{gathered}
N_{q}=\left(\lambda^{2} \sigma^{2}+\rho^{2}\right) / 2(1-\rho)(11) \\
N_{m}=\rho+N_{q}(12) \\
T_{q}=N_{q} / \mu \quad(13) \\
T_{m}=T q+1 / \mu=1 / \mu+\left(\lambda^{2} \sigma^{2}+\rho^{2}\right) /[2 \mu(1-\rho)]
\end{gathered}
$$

Para um dado tempo médio de serviço $1 / \mu, T_{m}$ cresce quando cresce o desvio-padrão. Ao se reduzir a variabilidade do tempo de processamento, também se reduz a espera por serviço. Podese associar $N$ e $T$ ao inventário e tempo de atravessamento de sistemas produtivos. Para redução de tempo de atravessamento, pode-se também reduzir a variabilidade dos processos e não apenas aumentar a capacidade média instalada. 


\subsection{Diagrama de Resultados}

Uma aplicação gráfica da teoria das filas é o diagrama de resultado (throughput diagram), como na Figura 2.

Figura 2: Diagrama de resultados



Fonte: Wiendahl (1995)

O diagrama é obtido acumulando-se, para um dado período de tempo, as entradas e saídas de ordens, em toneladas carregadas. As distâncias vertical e horizontal em $t$, entre entradas e saídas, correspondem ao inventário e ao tempo de atravessamento instantâneo. A inclinação média da curva de saída representa o desempenho do sistema, em toneladas por unidade de tempo (WIENDAHL, 1995). Como as ordens são discretas, têm-se saltos para os traçados de entrada e saída. Uma alternativa é substituí-los por retas equivalentes, obtidas por regressão linear. O coeficiente de determinação $R^{2}$ pode informar a qualidade da substituição: quanto mais próximo a 1 , melhor (SELLITTO, 2005). Para análise, geralmente é suficiente usar os valores médios do inventário e tempo de atravessamento e pela lei de Little, obter o desempenho médio $P_{m}(15)$, em toneladas por unidade de tempo (WIENDAHL; BREITHAUPT, 2001).

$$
P_{M}=I_{M} / T_{M}
$$

\section{Simulação computacional em operação logística}

A simulação computacional é uma técnica de Pesquisa Operacional que inclui a criação de um modelo computacional que representa uma parte do mundo real. Experimentos no modelo 
oferecem uma visão prévia do que acontecerá na realidade, com o objetivo de apoiar e reduzir a probabilidade de erros em decisões. Para Pidd (1998), simulação computacional consiste no uso de um modelo computacional para exploração de conhecimento e experimentação na realidade. A simulação permite testes what if: altera-se um ou mais parâmetros do modelo e observa-se o que acontece em um ou mais parâmetros de saída. Este tipo de teste possibilita que se antevejam os resultados de mudanças de porte antes que sejam feitas, evitando gastos e riscos desnecessários. Pode-se exigir uma solução de compromisso: o modelo deve ser simples o suficiente para ser manuseado e preciso o suficiente para ser útil. O modelo é submetido a entradas conhecidas, observando-se as saídas do modelo. As entradas podem ser consideradas fatores a vários níveis e as saídas a superfície de resposta do experimento. (SZYMANKIEWICZ et al., 1998).

Para Ballou (2006), a simulação computacional é uma técnica adequada para avaliar a dinâmica do fluxo de materiais e informações em operações logísticas. Por simulação computacional, a mais baixo custo e mais rapidez do que em experimentos reais, testam-se alternativas de abastecimento ou distribuição de produtos e verificam-se os possíveis resultados nos custos de produção e no nível de serviço. Para Lacerda (2000), os sistemas logísticos são um campo propício para a simulação computacional, pois incluem elementos que interagem entre si e são influenciados por fenômenos aleatórios, de difícil tratamento analítico. Para Cassel et al. (2002), um dos campos de aplicação da simulação é a logística de armazéns, fábricas e sistemas de distribuição, nos quais podem ser simuladas alternativas de layout, fluxos de pessoas e materiais, métodos de trabalho, meios de transporte e alocação de recursos.

Algumas características da aplicação apontam para o uso da simulação: (i) comportamento aleatório nos intervalos entre chegadas e tempos de carga; (ii) as operações se espalham no espaço, sendo difícil construir uma visão unificada de campo; e (iii) os rearranjos físicos dos equipamentos exigem paradas de máquinas e custos de instalação com experimentos não-garantidos (LAW; KELTON,1991). Os autores também apontam características genéricas do uso da simulação computacional que foram observadas na aplicação: (i) sistemas com variáveis aleatórias podem ser estudados pela simulação; (ii) a simulação controla melhor as variáveis intervenientes no experimento do que se controlaria no sistema real; (iii) simulam-se longos períodos em tempo reduzido; e (iv) a simulação não acha a solução ótima, mas testa alternativas.

Selltiz (1987) descreve a simulação computacional como método de pesquisa em ciências sociais aplicadas. Segundo a autora, a simulação ocupa uma terceira posição entre a experimentação de campo, na qual o pesquisador tem pouco controle, mas opera em verdadeira grandeza, e a experimentação de laboratório, na qual tem controle total, mas em grandeza reduzida. Na simulação, tem-se controle total em verdadeira grandeza, porém em um modelo virtual, não real. 


\section{A pesquisa}

A pesquisa ocorreu em uma fábrica de cimento, cujo processo de fabricação se divide em recebimento e processamento de matérias-primas e embalamento e despacho de produto acabado. A separação na fábrica é delimitada física e logicamente por silos, que operam como pulmão de produto acabado. O produto é fabricado para estoque, mediante uma lógica de produção empurrada e despachado sob encomenda, em produção puxada. A fabricação produz enquanto houver espaço nos silos e a expedição embala quando o veículo do cliente já se encontra na fábrica. A expedição pode ser em sacos de 25 e 50 kilogramas, por caminhões abertos, ou a granel, em caminhões fechados. Na Figura 3 se tem um diagrama lógico das duas etapas da fábrica, na qual se percebe o papel de pulmão separador dos silos.

O objetivo deste trabalho inclui a expedição de cimento ensacado. A expedição de cimento a granel dispõe de equipamento específico, que inclui uma balança rodoviária, e não interfere na expedição de cimento ensacado. A parte inicial da fábrica já foi objeto de estudo em Sellitto (1999) e corresponde ao trecho antes dos silos de cimento na Figura 3. A presente pesquisa corresponde aos trechos após os silos.

Figura 3: Estrutura logística interna do cenário da aplicação

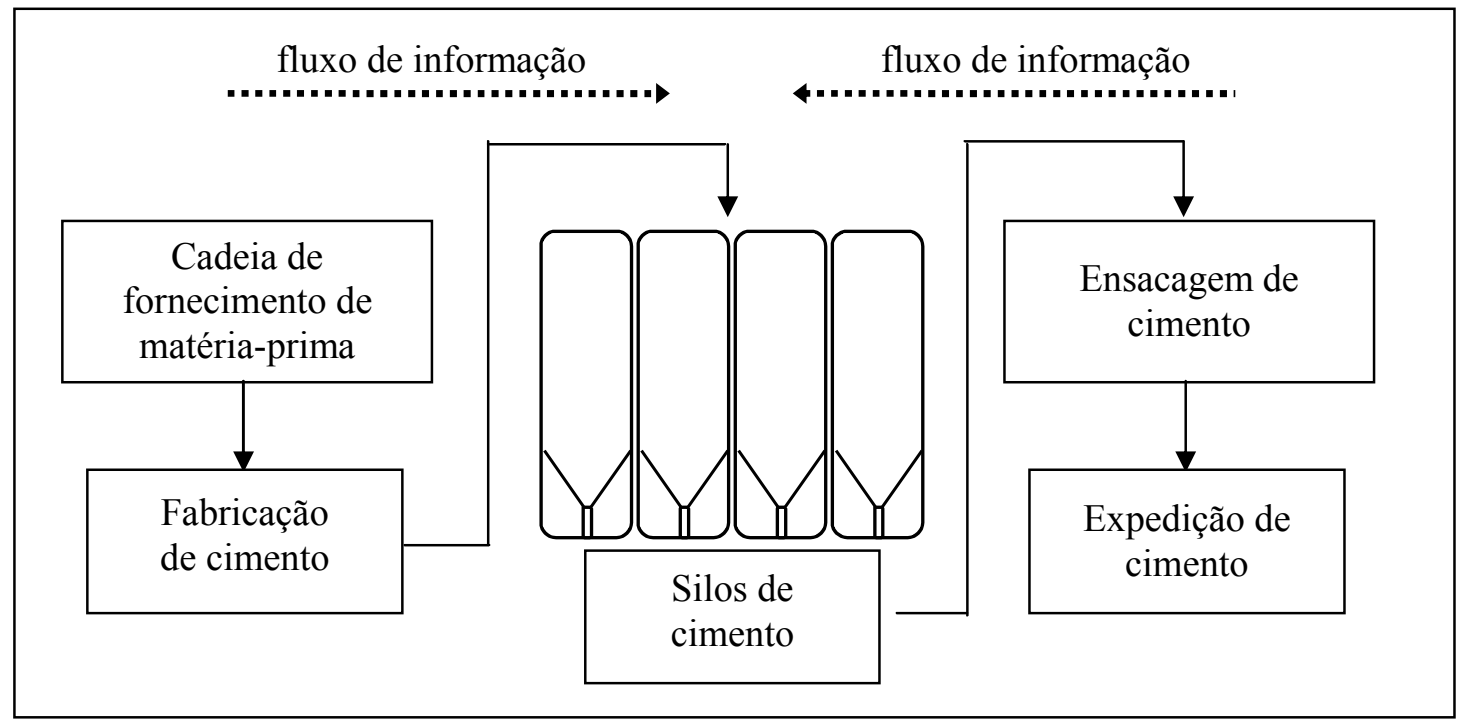

O método de trabalho foi: (i) descrição da operação; (ii) definição do objetivo do estudo, das alternativas e dos indicadores de comparação; (iii) levantamento de dados de realidade e ajuste de distribuições; (iv) desenvolvimento de um modelo para simulação em computador; (v) teste das alternativas; (vi) cálculo de parâmetros pela teoria das filas e diagrama de resultados; e (vii) análise das alternativas e conclusão. 


\subsection{A operação, o objetivo de pesquisa, as alternativas}

A descrição da operação foi obtida por observação participante e entrevistas.

Os clientes são lojistas revendedores de material de construção e compram a carga por telefone ou internet, especificando a tonelagem, o tipo de embalagem (sacos de 25 ou $50 \mathrm{~kg}$ ) e se tem transportador próprio ou contratará autônomo. Um funcionário da empresa designa cargas a transportadores e programa o dia da carga, em um horizonte de cinco dias. A ordem de entrada no dia é por ordem de chegada. Iniciado o turno, o operador de balança rodoviária autoriza a primeira entrada em grupos de quatro veículos para cada baia. A partir daí, uma nova entrada só é admitida com uma saída, mantendo no máximo quatro veículos em cada baia. Dois são atendidos e dois promovem a troca rápida no carregamento, para preservar a produtividade. Esta prática mantém controlado o inventário dentro da fábrica, ao redor de oito veículos. A balança é compartilhada com a pesagem de matéria-prima para a fabricação. Chegando matéria-prima, a entrada para carga é interrompida.

Os veículos são pesados em vazio (tara) e direcionados a duas baias de carregamentos, uma on-line automática (ensacagem e carregamento simultâneos), e uma off-line automática (pequeno estoque ensacado à noite e armazenado em paletes). O veículo é carregado, volta para a portaria e pesado com carga. Pela diferença entre carga e tara, é emitida a nota físcal e o veículo é liberado para partir. Os transportadores preferem carregar o mais cedo possível, pois podem tentar uma nova carga à tarde. Tal fato gera acúmulo de veículos pela manhã. Seria conveniente que houvesse uma alternativa para acelerar o carregamento antes das $8 \mathrm{~h} 30$ minutos da manhã, pois transportadores que voltam garantem nova venda no mesmo dia.

O objetivo global da pesquisa foi identificar ao menos uma alternativa que pudesse reduzir o tempo médio entre a chegada de um veículo ao pátio da fábrica e sua saída carregado. A organização dos recursos de operação é como na Figura 4.

Figura 4: Alternativas de expedição de cimento ensacado

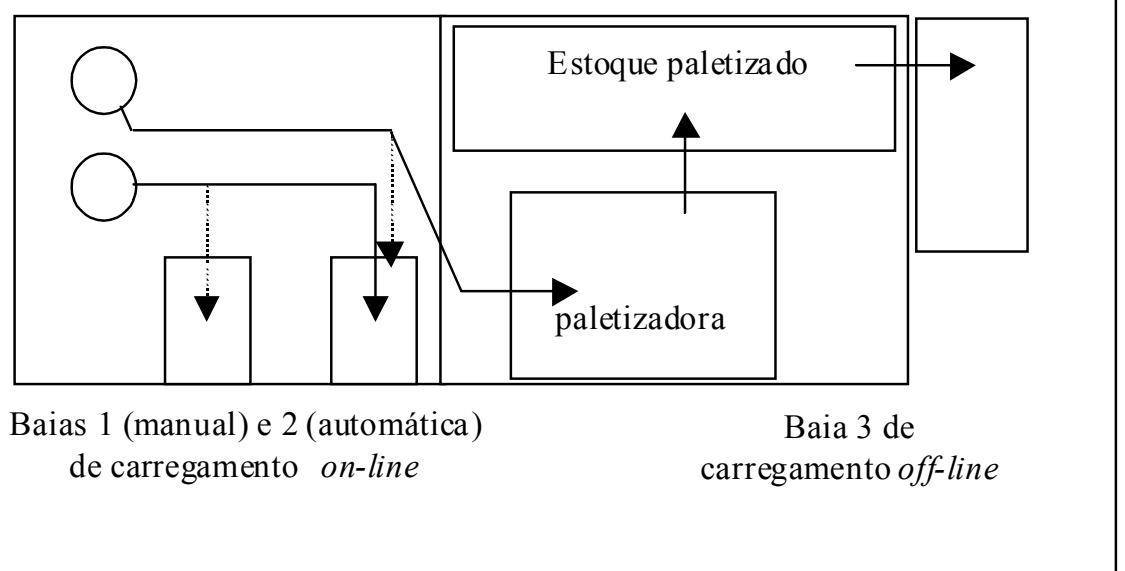


Foram consideradas as conseqüências da alternativa quanto à ocupação dos recursos. Os indicadores de apoio à decisão foram: (i) taxa de saída, em ton/min; (ii) ocupação de recursos; (iii) tempo médio de permanência de veículo no sistema, em minutos; (iv) variabilidade do tempo de permanência; e (v) inventário médio de ordens de carregamento, equivalente ao tamanho da fila. As alternativas de controle são: (i) o deslocamento da operação de entrada de matéria-prima da balança de entrada para balança secundária e (ii) reativação da baia B1 manual. Ambas valem para o período de $6 \mathrm{~h}$ às $8 \mathrm{~h} 30 \mathrm{~min}$ da manhã. No segundo caso, as baias 1 e 2 são alimentadas pelas máquinas ensacadeiras durante o período. Ao fim da tarde, uma das ensacadeiras faz estoque paletizado. Durante as duas horas e meia, operam as três baias simultaneamente.

\subsection{Levantamento de dados e cálculo de parâmetros}

Observou-se que a taxa de chegada de veículos ao pátio é maior entre $6 \mathrm{~h}$ e $8 \mathrm{~h} 30 \mathrm{~min}$, caindo após este horário. Após as 16h, a taxa chega quase a zero. Foram coletados dados entre $6 \mathrm{~h}$ e 8h30min de sessenta dias de operação. Os fatores de aleatoriedade foram os intervalos entre chegadas e o tempo de carregamento.

Os intervalos entre chegadas e entre entradas são calculados diminuindo-se os horários dos registros consecutivos das requisições de carga e de entrada na portaria. Os tempos de carregamento são a soma dos tempos: (i) acesso do veículo à baia; (ii) preparação e carga, em tonelagens variáveis; (iii) perdas de tempo por mau funcionamento durante a carga; (iv) colocação manual de lona e preparação para a viagem; e (v) acesso à balança, pesagem final e extração da nota fiscal.

Os dados foram analisados com a ajuda do software Stat:fit. O software seleciona automaticamente as distribuições mais prováveis, ajusta-os e classifica os ajustes (rank) conforme um cálculo não especificado na ajuda. Distribuições exponenciais não foram rejeitadas para as chegadas no pátio e entradas na fábrica, e por conseqüência as saídas. Portanto, os processos de chegada e de serviço são processos de Poisson. As distribuições mais bem ajustadas aos dados são apresentadas na Tabela 1. A mesma foi construída a partir de relatório emitido pelo software. 
Tabela 1: Ajustes de distribuições de interesse

\begin{tabular}{cccc}
\hline variate & distribution & rank & acceptance \\
\hline intervalo entre chegadas no pátio & exponential $(3.33)$ & 100 & do not reject \\
intervalo entre entradas na fábrica & exponential $(4.48)$ & 100 & do not reject \\
carga por veículo em toneladas & normal $(19.81,5.08)$ & 100 & do not reject \\
tempo de espera para liberar & normal $(42.75,20.86)$ & 100 & do not reject \\
tempo de carregamento on-line & lognormal $(42.5,2.43,0.802)$ & 78.0 & do not reject \\
& exponential $(45.0,12.6)$ & 78.0 & do not reject \\
& normal $(57.6,10.5)$ & 73.8 & do not reject \\
tempo de carregamento off-line & lognormal $(31.9,2.8,0.744)$ & 99.4 & do not reject \\
& exponential $(36 ., 16.9)$ & 73.3 & do not reject \\
& normal $(52.9,13.4)$ & 25.7 & do not reject \\
& uniform $(36,77)$ & 4.37 & do not reject \\
\hline
\end{tabular}

A carga se distribui segundo uma normal $N(19,81 ; 5,08)$ toneladas por caminhão. Os tempos de espera, por compartilhamento de balança, entre a chegada no pátio e a liberação para entrada na fábrica se ajustaram a uma distribuição normal $N(42,75 ; 20,86)$. Os tempos de carregamento on-line e off-line se ajustaram a várias distribuições, entre as quais as exponenciais de média 45 e 36 minutos por carga e deslocamentos temporais de 12,6 e 16,9 minutos, respectivamente. A diferença se deve à tecnologia adotada. No carregamento on-line, há empilhamento automático saco a saco no caminhão. No carregamento off-line, o cimento já foi paletizado e a operação requer apenas empilhadeira. A não-rejeição da exponencial indica dois sistemas $\mathrm{M} / \mathrm{M} / 1$.

\subsection{Análise das alternativas}

A operação logística não é satisfatória no período, pois a fila não converge a um valor finito e é crescente. As alternativas propostas para teste foram deslocar a entrada de matéria-prima para outra balança e também carregar manualmente em B1. Na primeira, foram eliminados o compartilhamento de balança e a espera para entrada na fábrica. O sistema de fila passa a ser representado por uma taxa de chegada de veículos no pátio e uma taxa de serviço, que se reflete na taxa com que veículos são admitidos na fábrica (um veículo entra se outro sai). Para a segunda alternativa, foi admitido que as taxas de serviço de B1 e B2 são iguais, pois não há dados de B1.

Para o teste, foi modelado o sistema em computador. Pela simplicidade dos casos, foi usada a planilha eletrônica Excel ${ }^{\circledR}$. Sellitto (2005) estudou o uso da planilha como simulador, comparando o desempenho do gerador randômico com exigências apontadas por Pidd (1998). O autor concluiu que a planilha é satisfatória quando as exigências de relatórios e de lógicas de intertravamento são leves, como ocorre no caso. Mesquita e Goldenberg (2006) também usaram planilhas em operações logísticas internas em manufatura. Os autores apresentam mais argumentos, referenciando outros autores, sobre o uso de planilhas para projetos de simulação de menor porte. 
A lógica de simulação foi: os intervalos entre chegadas no pátio seguem uma exponencial de média 3,33 minutos. O compartilhamento da balança e do balanceiro com a entrada de matériaprima impõe uma espera entre a chegada no pátio e a disponibilidade para entrada. A balança é ocupada em cerca de $35 \%$ do tempo com a pesagem de matéria-prima. O veículo chega e estaciona no pátio. O motorista pega uma ficha e vai a uma sala de espera, com display de chamada. Chamada sua ficha, o motorista se dirige à balança e recebe a designação de carga e baia e entra na fila liberada para entrar na fábrica. A partir deste momento, a entrada ocorre quando a fábrica requisitar um novo veículo e a balança estiver liberada.

A Figura 5 representa o sistema simulado.

Figura 5: Representação do sistema simulado

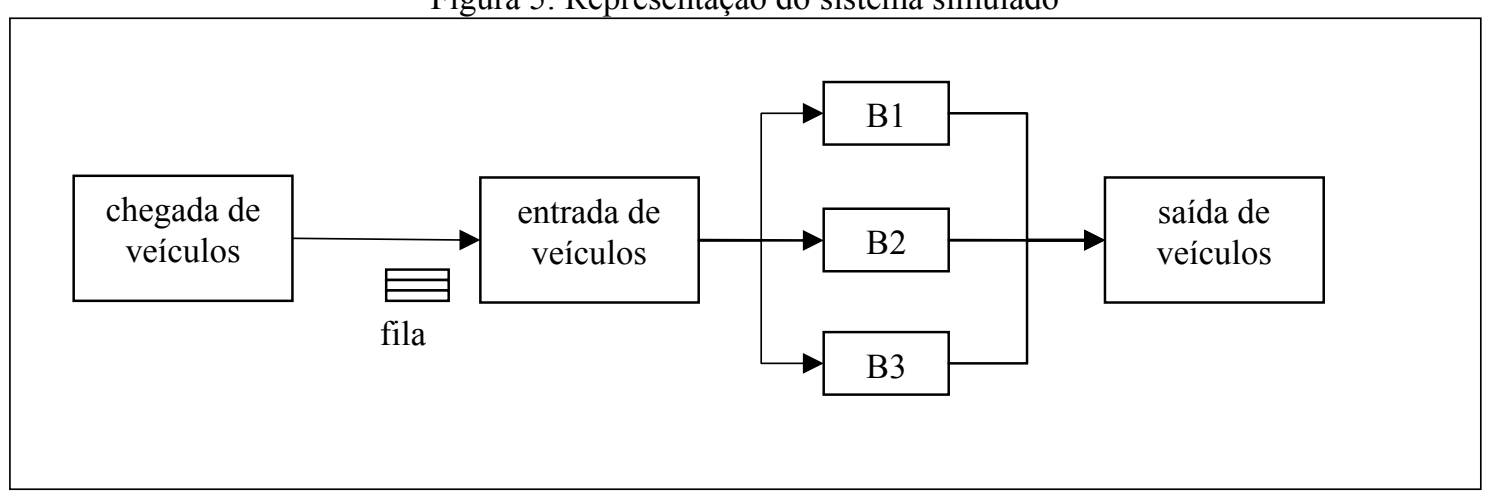

Há restrições na relação baia-veículo. Veículos maiores e em melhor estado de conservação são direcionados a B3. Veículos pequenos ou em mau estado são direcionados a B1 ou B2. Na entrada, o veículo é tarado (pesado em vazio) e na volta é pesado cheio. A diferença consta na nota fiscal e é faturada. Quanto à matéria-prima, o veículo é pesado cheio na entrada e vazio na saída e a diferença consta na nota fiscal a pagar pela empresa. Só há uma fila no sistema, entre a chegada no pátio e a entrada na fábrica. Devido à lógica de quatro veículos por baia (dois em carregamento e dois para troca rápida), não há filla dentro da fábrica. A troca rápida faz parte da operação e o sistema pode ser considerado de canal único a três servidores.

Admitidos os modelos exponenciais para o atendimento, e como os veículos são designados às baias antes da entrada, é possível tratar o sistema de filas como três $M / M / 1$. Também pode-se considerar o total de operações na fábrica como uma grande operação, cujo reflexo são as solicitações de mais veículos à portaria, mensuradas pela taxa de saída, igual à taxa de entrada, pois uma entrada é autorizada por uma saída. Por tal argumentação, chega-se a um sistema M/M/1, cujo formulário é mais simples. 
Foram simuladas quarenta e oito chegadas de caminhões (duas horas e meia a três minutos entre caminhões, múltiplo de quatro caminhões por baia). A primeira rodagem do modelo replicou a situação atual da fábrica. As demais replicaram a primeira e a segunda alternativa.

$\mathrm{Na}$ primeira alternativa, eliminou-se o compartilhamento da balança. A portaria possui uma pista lateral livre, pela qual passariam os veículos de matéria-prima, direcionados a uma balança existente dentro da fábrica. Seria necessário designar um balanceiro específico para esta balança, que teria pequena ocupação. Os gestores entrevistados acreditam que seria desnecessário entregar fichas, pois a menor ocupação do balanceiro faria com que a fila fosse pequena. Tal fato já ocorre hoje, à tarde. Tão logo o veículo chega no pátio, o motorista recebe a autorização e a entrada depende apenas da requisição de um novo veículo pela fábrica. Na segunda alternativa, uma terceira baia, igual à segunda, foi reativada.

O resultado da simulação surge na Tabela 2. A tabela apresenta as taxas de entrada (a mesma para os três casos) e de saída, a ocupação, a média e o coeficiente de variação (desviopadrão dividido pela média) para o tempo de permanência e o cálculo do inventário pela lei de Little, obtido multiplicando a primeira e a quarta colunas para as três situações. Foram feitas dez replicações para cada caso.

Tabela 2: Resultados da simulação

\begin{tabular}{rcccccc}
\hline & $\begin{array}{c}\text { Taxa de } \\
\text { entrada } \\
\text { (ton/min) }\end{array}$ & $\begin{array}{c}\text { Taxa de } \\
\text { saída } \\
\text { (ton/min) }\end{array}$ & $\begin{array}{c}\text { Ocupação } \\
\text { do sistema } \\
\text { logístico }\end{array}$ & $\begin{array}{c}\text { Tempo } \\
\text { médio de } \\
\text { permanên- } \\
\text { cia (min) }\end{array}$ & $\begin{array}{c}\text { cv do } \\
\text { tempo }\end{array}$ & $\begin{array}{c}\text { Inventário } \\
\text { médio (ton) }\end{array}$ \\
\hline Atual & 6,69 & 2,71 & 2,42 & 144 & 0,38 & 963 \\
$1^{\text {a }}$ alternativa & 6,69 & 2,51 & 2,67 & 136 & 0,45 & 970 \\
$2^{a}$ alternativa & 6,69 & 3,34 & 2,00 & 91 & 0,53 & 608 \\
\hline
\end{tabular}

As Figuras 6, 7 e 8 apresentam os diagramas de resultados para a situações atual, primeira e segunda alternativa. Os valores dos $R^{2}$ corroboraram os diagramas. Pelas figuras, é possível confirmar que as entradas sempre superaram as saídas no período, ou seja, mesmo que se adote a segunda alternativa, para equilibrar o sistema será necessário contingenciar a chegada de veículos naquele horário. 
Figura 6: Diagrama de resultados da situação atual

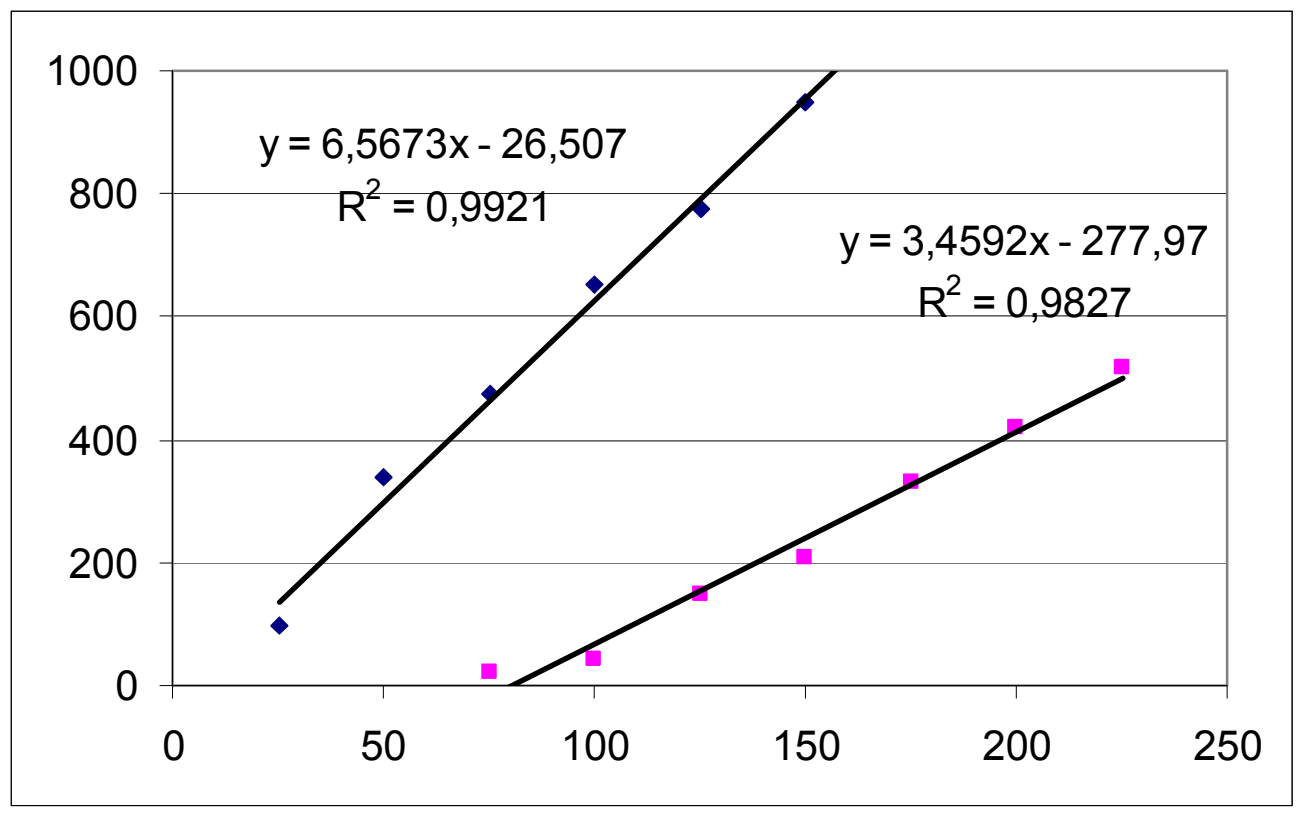

Figura 7: Diagrama de resultados da primeira alternativa

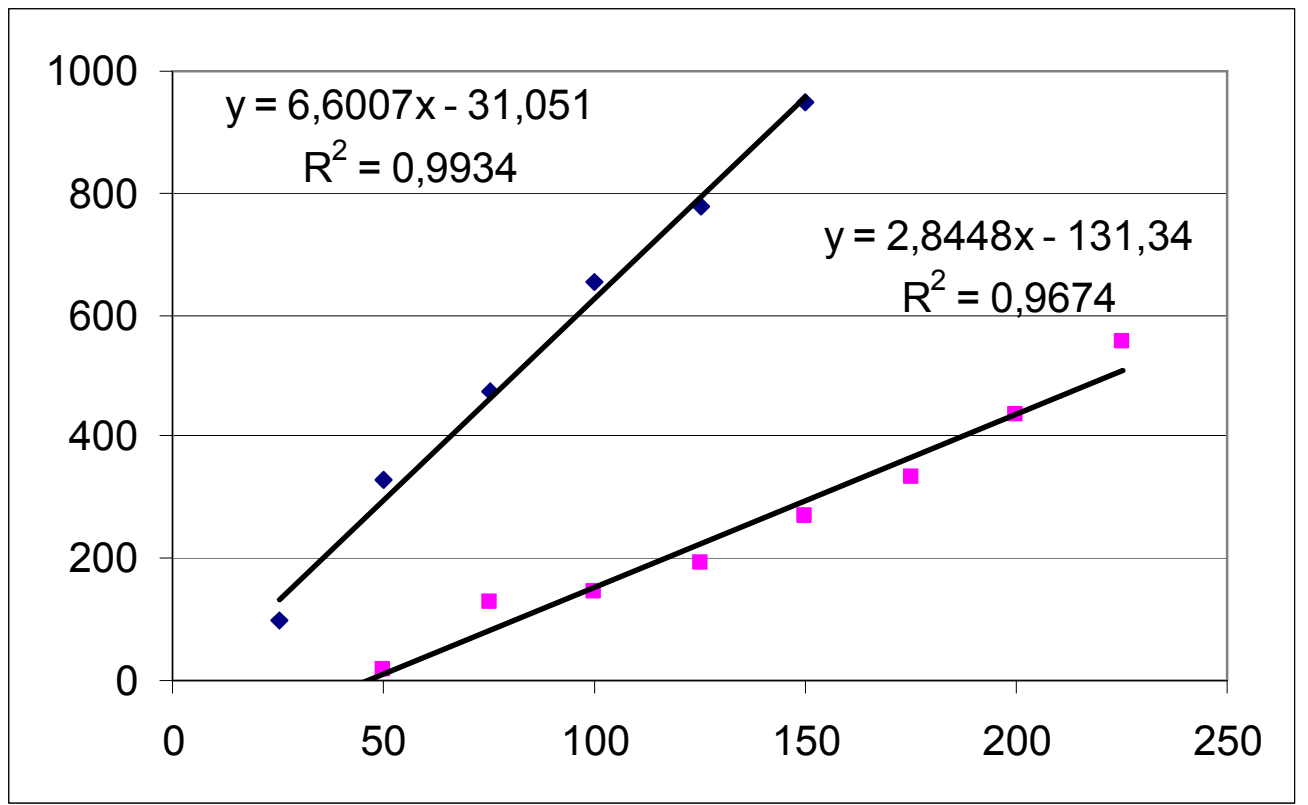


Figura 8: Diagrama de resultados da segunda alternativa

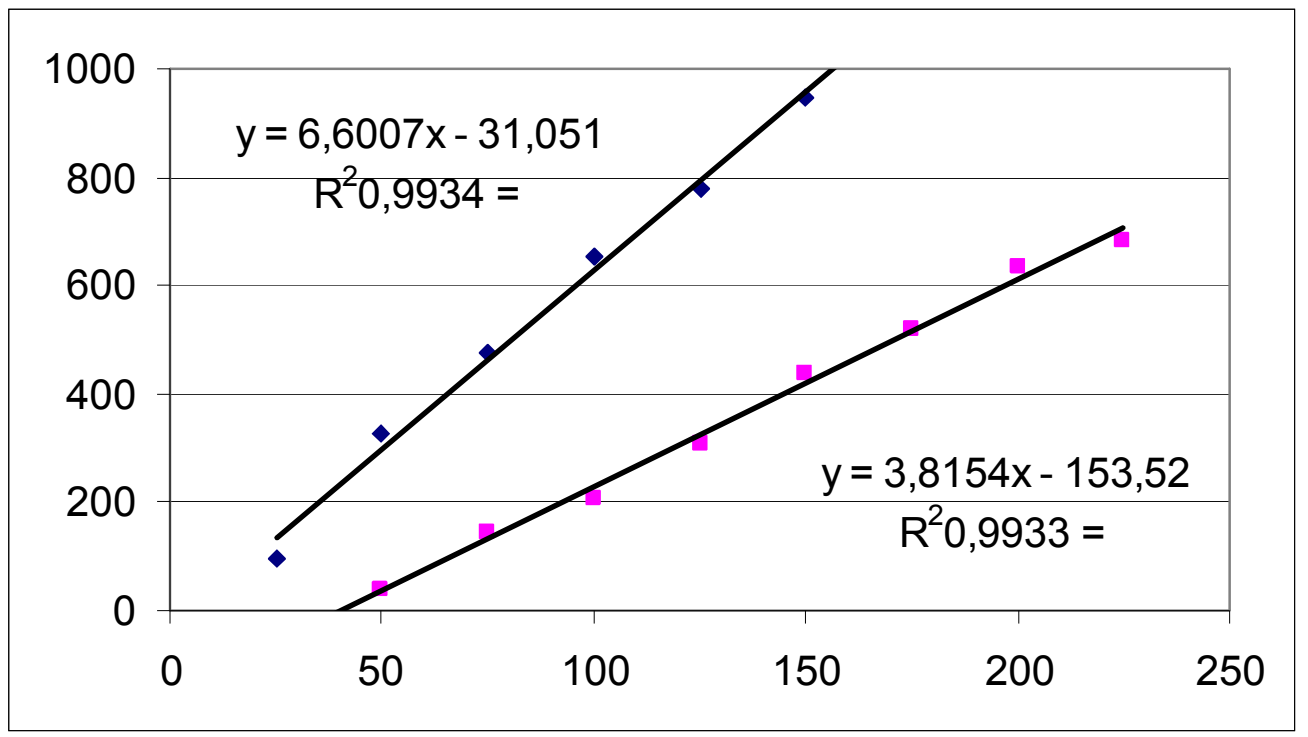

Os resultados específicos para cada baia surgem na Tabela 3. Observa-se que B3 tem melhor resultado em taxa de saída, tempo de permanência e inventário. No entanto, apresenta mais variabilidade do que B2 e B1-B2. Esta variabilidade aumentada não era esperada e pode ser tema de futuras investigações.

Tabela 3: Resultados separados por baias

\begin{tabular}{rcccccc}
\hline & $\begin{array}{c}\text { Taxa de } \\
\text { entrada } \\
\text { (ton/min) }\end{array}$ & $\begin{array}{c}\text { Taxa de } \\
\text { saída } \\
\text { (ton/min) }\end{array}$ & $\begin{array}{c}\text { Ocupação } \\
\text { do sistema } \\
\text { logístico }\end{array}$ & $\begin{array}{c}\text { Tempo } \\
\text { médio de } \\
\text { permanên- } \\
\text { cia (min) }\end{array}$ & $\begin{array}{c}\text { cv do } \\
\text { tempo }\end{array}$ & $\begin{array}{c}\text { Inventário } \\
\text { médio (ton) }\end{array}$ \\
\hline Atual B2 & 3,65 & 1,57 & 2,56 & 174 & 0,31 & 635 \\
Atual B3 & 3,52 & 1,77 & 2,52 & 116 & 0,35 & 408 \\
$1^{\text {a }}$ alternativa B2 & 3,65 & 1,34 & 2,81 & 171 & 0,37 & 624 \\
$1^{\text {a }}$ alternativa B3 & 3,52 & 1,66 & 2,79 & 99 & 0,40 & 348 \\
$2^{a}$ alternativa B1-B2 & 4,77 & 2,21 & 2,15 & 108 & 0,43 & 515 \\
$2^{a}$ alternativa B3 & 2,61 & 1,67 & 1,56 & 54 & 0,46 & 140 \\
\hline
\end{tabular}

Incerteza na demanda, variabilidade nas trocas de lotes e desempenho baixo nas cadeias produtivas e logísticas são fatores que dificultam a redução de custo e colocam em risco o cumprimento de datas-devidas. Sob certas circunstâncias, os objetivos logísticos de clientes e de fabricantes podem ser contraditórios, sendo necessária uma solução de compromisso: uma configuração tal que reduza os tempos de atravessamento sem prejudicar demasiadamente a ocupação do sistema produtivo. Nos casos em que há preparação de máquinas, ao se aumentar o lote de produção, reduzindo a perda por preparação, aumenta-se o inventário em processo, criando uma fila de espera para as ordens, o que retarda o atendimento aos novos pedidos. Ao diminuir o lote, por variabilidade nas entradas, é possível que baixe a ocupação da fábrica. 


\section{Discussão}

Seguem considerações sobre os resultados e o método de pesquisa. Inicia-se justificando o uso da simulação.

A taxa de entrada $\lambda$ é de 6,69 toneladas por minuto. As taxas de saída $\mu$, para os três casos, são de 2,71; 2,51 e 3,34 toneladas por minuto. Os tempos médios de permanência são, respectivamente, 144, 136 e 91 minutos. A justificativa para a menor taxa de saída com menor tempo médio do segundo caso é que o não compartilhamento da balança permitiu que a expedição começasse mais cedo e se espalhasse mais uniformemente no período. O tempo médio é menor porque não faltou carga nas baias. A taxa de saída é menor porque as primeiras saídas ocorreram mais cedo e o cálculo da taxa de saída é iniciado no momento da primeira carga.

Foi apontado que é possível considerar a operação total como $\mathrm{M} / \mathrm{M} / 1$, valendo o formulário da teoria das filas. No entanto, a aplicação das equações não foi viável, pois $\lambda>\mu \mathrm{em}$ todos os casos. O tamanho da fila, e por conseqüência o tempo de permanência, não convergem a valores finitos. Havia expectativa de que uma das alternativas pudesse tornar $\mu>\lambda$, o que justifica a inclusão do formulário na pesquisa.

Justificou-se o uso a planilha ao invés de simuladores específicos por o gerador randômico ter se mostrado superior às exigências listadas em Pidd (1998), os comandos de intertravamento serem simples e a análise de resultados ter sido feita pelo diagrama de resultados. $\mathrm{O}$ uso de simulador específico teria requerido esforço para o domínio dos comandos específícos. $\mathrm{O}$ uso da planilha permitiu que o esforço de pesquisa fosse concentrado no caso, não na ferramenta.

Os diagramas de resultados mostram traçados que se afastam, confirmando a tendência de aumento de inventário ao longo do tempo, mesmo no melhor caso. À medida que se avança no tempo, o inventário médio e o tempo médio de atravessamento crescem. O desequilíbrio requer uma ação de controle. As alternativas são: (i) reforço de capacidade de carga; (ii) redução na demanda instantânea; ou (iii) ambas. Em função da ausência de um instrumento concentrado de medição das variáveis relevantes, é possível que a operação só se aperceba do desequilíbrio quando uma condição de contorno física, como a capacidade de estacionamento, seja ultrapassada.

A eliminação do compartilhamento da balança mostrou-se pouco promissora, pois a redução no tempo de carga foi pequena. Esta conclusão surpreendeu, pois os gestores entrevistados acreditavam que esta seria uma importante causa de atraso no carregamento. É possível que o engano tenha sido causado pela dificuldade de um gestor avaliar todas as variáveis ao mesmo tempo, pois as implicações do comportamento das mesmas são distribuídas no tempo e no espaço. Neste caso, um instrumento concentrador de informações, tal como o diagrama de resultado, 
composto pelas fases da fila, pode ser útil, pois informa imediatamente que fase do sistema está sobrecarregada e, portanto, travando a taxa de saída.

A alternativa mais promissora foi a reativação da baia manual B1, porém não foi capaz de equilibrar o sistema, apenas reduzir o desequilíbrio, pois a ocupação, se bem que reduzida, continuou maior do que 1. Caso a empresa decida que quer equilíbrio, então deve restringir a entrada de veículos no horário, de modo que o diagrama de resultados forme duas retas paralelas. A distância horizontal entre as retas é o inventário que surge na operação. A distância vertical é o tempo de permanência. O inventário deve ser calibrado no menor valor tal que, mesmo com variabilidade, não haja interrupção significativa por falta de carga. Sellitto (2005) chama este processo de construção da curva logística e o inventário calibrado de inventário crítico. A curva logística pode ser obtida da simulação e considera a taxa de saída do sistema.

Para três baias, a taxa de saída em veículos é de $[3,34$ ton/min $] /[19,81$ ton/veículo] $=$ [0,168 veículo/min]. Seja que a empresa decida contingenciar a demanda, admitindo um certo número de veículos $n$ a cada meia hora. A Tabela 4 apresenta as probabilidades de que a fábrica requisite até $n$ veículos em meia hora, calculadas por um modelo de Poisson de média 0,168 requisições por minuto. Por exemplo, a chance de a fábrica requisitar até seis veículos no período é de $75,6 \%$ e até dez é $98,6 \%$.

Tabela 4: Probabilidades de até $n$ requisições de veículos em meia hora

\begin{tabular}{cc}
\hline $\boldsymbol{n}$ & $\boldsymbol{P}(\boldsymbol{n})$ \\
\hline 6 & $75,6 \%$ \\
7 & $86,2 \%$ \\
8 & $92,9 \%$ \\
9 & $96,7 \%$ \\
10 & $98,6 \%$ \\
11 & $99,4 \%$ \\
12 & $99,8 \%$ \\
\hline
\end{tabular}

Seja a seguinte política de admissão ao pátio: a primeira meia hora inicia com onze veículos; nas próximas, admite-se o número que falta para onze veículos. A probabilidade de não faltar veículo quando requisitado é de 99,4\%. Tal política manteria controlado o tamanho da fila e, por conseguinte, o tempo total de permanência de veículos na operação, correndo um risco de menos de $0,5 \%$ de quebra de serviço.

O número médio de requisições em meia hora será [0,168 veículos/minuto x 30 minutos] 5 veículos. Seis veículos formariam o pulmão de proteção da fila contra variabilidades. O máximo tempo de espera para entrar na fábrica é $[11$ veículos x $1 / 0,168$ minuto/ veículos] $\cong 65$ minutos. A fila média é de $[5+6 / 2]=8$ veículos. O tempo médio de espera é de $[8$ veículos x 1/0,168 minuto/ 
veículos $] \cong 48$ minutos. Por raciocínio análogo, se a empresa decidisse que a fila máxima será de oito veículos, a chance de sucesso baixa para $\cong 93 \%$, a fila média baixa para 6,5 e o tempo médio de espera na fila baixa para 39 minutos. A definição do tamanho ótimo da fila leva em consideração os custos da espera e da quebra de serviço e é remetida à continuidade.

A simulação apontou os benefícios da alternativa preferencial, a reativação de B1, mas há ônus gerenciais e operacionais que devem ser examinados.

É necessário estoque prévio de cimento paletizado, pois, por duas horas e meia, B3 operará sem abastecimento das ensacadeiras. Geralmente, à tarde, a taxa de entrada cai, sendo este o momento de interromper a carga por B3, que tem o melhor desempenho, e fazer estoque. Será necessário decidir a que momento interromper B3. Esta decisão deve levar em conta a previsão de entrada para o resto da tarde, o estoque remanescente do dia, originado de eventuais diferenças entre as taxas de produção e de entrega, e o risco de insatisfazer os clientes do momento, pois vão carregar em baia de menor desempenho por motivos que não o beneficiarão. Esta decisão deve reunir interesses que podem ser contraditórios, tais como a supervisão logística e comercial, que quer rapidez para o cliente (não libera B3, só faz estoque remanescente e opera com baixa produtividade nas duas baias), e de produção, que quer eficiência (concentra a carga em B2, garante produtividade e penaliza os clientes da tarde).

Operacionalmente, há a necessidade de montar equipe de quatro carregadores manuais que trabalharão em torno de duas horas e meia por dia, das $6 \mathrm{~h}$ às $8 \mathrm{~h} 30 \mathrm{~min}$.

Um problema é o horário inicial. A empresa já antecipou o início do carregamento das $7 \mathrm{~h} 30 \mathrm{~min}$ para as $6 \mathrm{~h}$ em função da preferência do cliente em começar mais cedo para poder voltar no mesmo dia, como já mencionado. Como B2 e B3 são automatizados, a exigência de operadores é menor, um por baia, e a chegada dos mesmos é mais simples. Com B1, será necessária a chegada de quatro operadores, o que pode ser mais difícil naquele horário e provavelmente requererá transporte exclusivo.

Outro problema é o horário parcial. Não é vantajosa a contratação da equipe como funcionários, pois não cumpririam a jornada diária, e não há operadores que possam ser realocados, pois a fábrica é totalmente automatizada. A opção é a contratação de terceiros por tempo parcial, junto a empresa locadora de mão-de-obra ou cooperativa, que se responsabilizaria pela gestão da equipe, incluindo direitos sociais, transporte, segurança, treinamento e bem-estar dos operadores.

Em resumo, a pesquisa ofereceu uma conclusão. Se B1 for ativada entre as $6 \mathrm{~h}$ e as 8h30min, o tempo médio de permanência de veículos no sistema de carregamento cai de 144 minutos para 91 minutos, mas a empresa deve contratar uma equipe de quatro operadores, administrados por locadora de serviços profissionais. O trade-off é: uma redução de 53 minutos no 
tempo médio de permanência vale o custo da contratação da equipe mais o ônus de uma decisão diária sobre que horário interromper a carga por B3?

Incerteza na demanda, variabilidade nas trocas de lotes e desempenho baixo nas cadeias produtivas e logísticas são fatores que dificultam a redução de custo e colocam em risco o cumprimento de datas-devidas. Sob certas circunstâncias, os objetivos logísticos de clientes e de fabricantes podem ser contraditórios, sendo necessária uma solução de compromisso: uma configuração tal que reduza os tempos de atravessamento sem prejudicar demasiadamente a ocupação do sistema produtivo. Nos casos em que há preparação de máquinas, ao se aumentar o lote de produção, reduzindo a perda por preparação, aumenta-se o inventário em processo, criando uma fila de espera para as ordens, o que retarda o atendimento aos novos pedidos. Ao diminuir o lote, por variabilidade nas entradas, é possível que baixe a ocupação da fábrica.

\section{Conclusão}

Este artigo apresentou uma pesquisa que testou a viabilidade de duas alternativas para redução do tempo de permanência de veículos em uma operação logística de carga e expedição de cimento ensacado entre 6h e 8h30min: a ativação de balança adicional, para desobstruir a entrada e a reativação de baia manual, desativada quando da troca da tecnologia. O ganho potencial é: reduzir o tempo médio de permanência de veículos na operação de 144 para 91 minutos. A decorrência da escolha é: contratação de equipe com quatro operadores, por meio de empresa locadora de mão-deobra, e acréscimo de uma decisão gerencial diária, o momento de interrupção de carga por B3.

A pesquisa abriu outras questões de pesquisa. Uma diz respeito à variabilidade aumentada observada na simulação de B3. Esta não era esperada e pode justificar uma nova pesquisa, pois, como demonstrado pela teoria das filas, acréscimo de variabilidade contribui para acréscimo de tempo médio. Se for possível identificar uma causa para esta variabilidade, será possível reduzir o tempo médio. Remete-se esta alternativa para trabalhos futuros. Outra é a construção da curva logística para a operação e a definição por cálculos econômicos do inventário ótimo, aquele tamanho de fila que minimiza a soma dos custos de espera e de interrupção na operação por falta de carga.

Quanto à técnica de simulação, não era objetivo de pesquisa o aprofundamento da técnica, mas talvez fosse importante repetir a aplicação com um simulador comercial e comparar os resultados. A planilha representou importante redução de tempo de pesquisa. O modelo tomou menos de doze horas para construção. O tempo total de projeto, incluindo instalação e treinamento em simulador, dificilmente seria menor. 
Também se remete para trabalhos futuros a aplicação completa da metodologia em outros tipos de sistemas logísticos, como operações de armazenagem e distribuição. Conclui-se colocando que o diagrama de resultados da teoria das filas, associado à simulação computacional parece representar alternativa promissora para medição e controle de sistemas logísticos, pois, quando a operação é distribuída no espaço, algumas vezes, pode ser difícil gerenciar os inventários e atrasos exclusivamente de modo visual e do chão-se-fábrica, sendo útil uma ferramenta informacional que sintetize as operações em um instrumento unificado, tal como o diagrama de resultados.

\begin{abstract}
This paper describes an application of computer simulation with electronic worksheet, aiming to identify the better choice for reducing the lead-time for trucks in a queue system in a logistic operation. The operation is the load and dispatching of packed cement. Trucks arrive and form a waiting line for releasing to load. After released, the truck waits to be requested for loading, in one of two loading bays. Four vehicles are admitted in each bay, keeping controlled an inventory of eight vehicles inside the operation. The B3 bay loads from stock, with higher performance then the B2 bay, which loads online from packing machines. From 6:00 to 8:30 in the morning, the arrive rate is greater then the service rate, with crescent queue. The problem is to find an alternative in the process to reduce lead-time, with minimum lost of resource occupation. Three alternatives were tested in a simulation model with worksheet. Queue theory and through diagram were used to judge the simulation. The best choice was the reactivation, by two and a half hours each day, the B1 loading bay, operated by human operators, now off. The mean lead-time is reduced from 144 to 91 minutes.
\end{abstract}

Key-words: Simulation with worksheet, Simulation in logistics operations, Queue theory in logistics, Throughput diagram..

\title{
Referências
}

BALLOU, R. Gerenciamento da cadeia de suprimentos/logística empresarial. P. Alegre: Bookman, 2006

CASSEL, R.; CARMO, F.; CAMPANA, F.; RITTER, F.; SILVA, M. Simulação da logística interna da área de armazenagem de uma empresa do setor moveleiro. In. ENCONTRO NACIONAL DE ENGENHARIA DE PRODUÇÃO, 22., 2002, Curitiba. Anais... Curitiba: ABEPRO, 2002

CHATFIELD, D.; HARRISON, T.; HAYYA, J. SISCO: An object-oriented supply chain simulation system. Decision Support Systems. V.42, n.1, p.422-434, 2006.

cross ${ }^{\text {eef }}$

DISNEY, S.; NAIM, M.; TOWILL, D. Dynamic simulation modelling for lean logistics. International Journal of Physical Distribution \& Logistics Management. V.27, n.3/4, p.174-196, 1997.

cross ${ }^{\text {ref }}$

KLEINROCK, L. Queueing Systems, Volume I. N. York: John Wiley \& Sons, 1975

HILLIER, F.; LIEBERMAN, G. Introduction to operations research. Singapore: McGraw-Hill, 1995

LACERDA, L. Automação na armazenagem: desenvolvendo e implementando projetos de sucesso. In: FLEURY, P. (org.). Logística empresarial: A perspectiva brasileira. São Paulo: Atlas, 2000.

LAW, A.; KELTON, W. Simulation Modeling and Analysis. N. York: McGraw-Hill, 1991

MESQUiTA, M.; GOLDENBERG, D. Simulação em Planilhas de Linhas de Produção Puxada. In. ENCONTRO NACIONAL DE ENGENHARIA DE PRODUÇÃO, 26., 2006, Fortaleza. Anais... Fortaleza: ABEPRO, 2006. 
PAPADOPOULOS, H.; HEAVEY, C.; BROWNE, J. Queuing theory in manufacturing systems analysis and design. London: Chapman \& Hall, 1993.

PERSSON, F.; ARALDI, M. The development of a dynamic supply chain analysis tool-Integration of SCOR and discrete event simulation. International Journal of Production Economics, In Press, Corrected Proof, Available online 12 March 2007.

PIDD, M.: Computer simulation in management science. Chichester: John Wiley \& Sons, 1998

SELLITTO, M. Sistema de produção sincronizado: uma aplicação em processos produtivos de propriedade contínuos segundo a teoria das restrições. Dissertação de Mestrado, PPGEP, UFRGS, Porto Alegre, 1999.

SELLITTO, M. Medição e controle de desempenho estratégico em sistemas de manufatura. Tese de doutorado. PPGEP-UFRGS, P. Alegre: 2005.

SELLTIZ, C. Métodos de pesquisa nas relações sociais. S. Paulo: EPU, 1987.

SZYMANKIEWICZ, J.; MCDONALD, J.; TURNER, K. Solving Business Problems by Simulation. London: McGraw-Hill, 1998.

TERZI, S.; CAVALIERI, S. Simulation in the supply chain context: a survey. Computers in Industry. V.53, n.1, p.3$16,2004$.

cross ${ }^{\text {ref }}$

WIENDAHL, H.-P.: Load-oriented manufacturing control. Berlin: Springer-Verlag, 1995.

WIENDAHL, H.; BREITHAUPT, J. Automatic production control applying control theory. International Journal of Production Economics, n.63, p.33-46, 2001.

ZEE, D.; VORST, J. A Modeling Framework for Supply Chain Simulation: Opportunities for Improved Decision Making. Decision Sciences. V.36, n.1, p.65-95, 2005.

cross ${ }^{\text {ref }}$

\section{DADOS DOS AUTORES}

Nome completo: Miguel Afonso Sellitto

Filiação institucional: UNISINOS

Departamento: PPGEPS

Função ou cargo ocupado: Professor e pesquisador

Endereço completo para correspondência (bairro, cidade, estado, país e CEP): Av. Unisinos 950, São Leopoldo, 93022-000, RS, Brasil

Telefones para contato: 51 3591-1122

e-mail: sellitto@unisinos.br

\section{Nome completo: Miriam Borchardt}

Filiação institucional: UNISINOS

Departamento: PPGEPS

Função ou cargo ocupado: Professora e pesquisadora

Endereço completo para correspondência (bairro, cidade, estado, país e CEP): Av. Unisinos 950, 
São Leopoldo, 93022-000, RS, Brasil

Telefones para contato: 51 3591-1122

e-mail: miriamb@unisinos.br

Nome completo: Giancarlo Medeiros Pereira

Filiação institucional: UNISINOS

Departamento: PPGEPS

Função ou cargo ocupado: Professor e pesquisador

Endereço completo para correspondência (bairro, cidade, estado, país e CEP): Av. Unisinos 950, São Leopoldo, 93022-000, RS, Brasil

Telefones para contato: 51 3591-1122

e-mail:gian@unisinos.br

Recebido para publicação em: 05/10/2009

Aceito para publicação em: 02/12/2009 